\title{
Review
}

\section{New Progress in the Cause Analysis and Nursing of Respiratory Tract Infection after Abdominal Surgery under General Anesthesia}

Congxian Yang

Department of Anesthesiology, Shandong Provincial Hospital Affiliated to Shandong University, Jinan China

\author{
Keywords \\ general anesthesia; abdominal surgery; \\ respiratory tract infection; cause; nursing

\section{Correspondence} \\ Congxian Yang, \\ E-mail: Yangcx66@126.com \\ DOI: 10.1515/ii-2017-0135
}

\begin{abstract}
This article provides a review of the causes of respiratory tract infection after abdominal surgery. These causes include general anesthesia, intubation factors, factors inherent to the patient, surgical factors, the injudicious use of antimicrobial agents, and the environmental factors of the ward. The perioperative management of the respiratory tract should be strengthened. Health education, respiratory function training, oral nursing intervention, atomization inhalation, and personalized expectoration methods should receive more attention to decrease the complications and promote the early rehabilitation of patients after abdominal surgery.
\end{abstract}

Respiratory tract infection is a common complication after abdominal surgery. The incidence of lower respiratory tract infection after abdominal surgery is significantly higher under general tracheal intubation anesthesia than under other anesthesia methods ${ }^{[1]}$. Chinese scholars have reported that the rate of respiratory tract infection after tracheal intubation was $20.17 \%$ in China ${ }^{[2]}$. Therefore, the prevention of respiratory tract infections and the nursing of patients who underwent intubation for general anesthesia are crucial. This paper provides a review of the causes of and nursing methods for respiratory tract infection in patients who underwent abdominal surgery with general anesthesia.

\section{Causes of Respiratory Tract Infection}

\section{General anesthesia intubation factors}

1) Intubation for general anesthesia provides a direct connection between the bronchial tree and the external environment. The nose and oropharynx lose their function as the first line of defense against infection and cannot moisturize entering airflow. Thus, the respiratory tract becomes too dry. 2) Tracheal intubation for general anesthesia and a series of invasive operations damage the respiratory mucosa and villi, thereby causing local inflammation, weakening ciliary movement, and increasing secretions ${ }^{[3]}$.

\section{Factors inherent to the patient}

The age, nutritional status, and smoking status of the patient are also important factors that influence the risk of respiratory infections. 1) Wei Chengmin et al. ${ }^{[4]}$ believed that the incidence of respiratory tract infection in patients after general anesthesia is positively correlated with the age of the patient. 2) Duan Hongli et al. ${ }^{[5]}$ held that poor preoperative nutritional status or insufficient postoperative nutrient supply is a direct reason for the low resistance of the patient, thereby increasing the probability of infection. 3) The probability of infection among smokers is significantly higher than that among non-smokers. Approximately $38.2 \%$ of long-term smokers(those who smoke more than 20 cigarettes per day or for more than 20 years) experience post-surgery pulmonary infection, whereas only $12.5 \%$ of non-smokers experience postsurgery pulmonary infection ${ }^{[6]}$.

\section{Medical factors}

1) Numerous epidemiological surveys have shown that pathogens on the hands of medical care workers are 
implicated in hospital infection, and that hand hygiene is the most important part of hospital infection control ${ }^{[7]}$. 2) Zhu Huadong et al. ${ }^{[8]}$ reported that deep intubation, unskilled intubation, and the incomplete equalization of extubation are high-risk factors for respiratory infection after intubation for general anesthesia.

\section{Surgical factors}

1) The loss of body fluids during surgery, postoperative fasting, and oxygen uptake produce sticky sputum, which is difficult to discharge. 2) Patients do not attempt or fear physical activities because of postoperative pain, indwelling pipelines and monitoring equipment, physical weakness among elderly patients, and other reasons. Thus, their lung movements are limited, their cough reflexes are weakened, and their respiratory secretions are not easily discharged and instead flow down to lungs with gravity. These factors collectively cause lung infections ${ }^{[9]}$.

\section{Irrational use of antimicrobial agents}

The occurrence of pulmonary infection in the hospital is related with the imbalance of the body's normal flora. This imbalance results from the abuse of broad-spectrum antibiotics: the normal microflora in the oropharynx and stomach of patients are inhibited, causing the mass propagation of conditional pathogens and drug-resistant strains ${ }^{[10]}$. Bronchial or pulmonary infection may occur when bacteria or fungi are inhaled into lower respiratory tract.

\section{Ward environmental factors}

The main source of pollution in the ward is the patient's own secretions and excreta, as well as the pathogens introduced by visiting family members. Although ambient temperature is maintained and receives special focus, air circulation is ignored, thus aggravating air pollution. Aerosols carrying microbes spread with the flow of indoor air. In addition, the entry of people into the ward accelerates the spread of aerosol microbes, which threatens the respiratory health of patients [11].

\section{Nursing measure}

\section{Health education}

Upon hospital admission, the patients should be advised to quit smoking. During winter, patients should stay warm to prevent catching a cold. Oral and skin hygiene should be enhanced to avoid damage to oral mucosa and skin. Infected lesions should be actively treated. Preoperative dietary advice should be given to patients to ensure adequate nutrition. In addition, patients should receive vitamin supplements to enhance their tolerance to surgery and infections ${ }^{[12]}$.

\section{Respiratory function training}

Exercises for respiratory function can improve the patient's lung function, improve the patient's tolerance to upper abdominal surgery, and reduce postoperative pulmonary complications ${ }^{[13]}$. Respiratory training methods include: 1) Deep breathing exercise: Thoracic breathing should be practiced after abdominal surgery. The patient places their hands on their sixth rib immediately under the armpit. While inhaling, the patient tightly closes their lips and deeply inhales through the nose. Their hand should feel the outward expansion of their chest. While exhaling, the patient pouts their lips into a whistle-like "O", allowing air to pass slowly through their narrowed lips. The patient inhales for $2 \mathrm{~s}$. The duration of exhalation is gradually increased to $10 \mathrm{~s}$. This method is practiced thrice a day for 10-15 min each time until surgery. 2) Effective cough training: The patient should first breathe deeply 5-7 times. The patient should keep their mouth open toward the end of inhalation. The patient should press their abdomen with their hands while coughing, slightly bend their shoulder inward, slightly tilt their head downward, and cough continuously several times.

\section{Oral care intervention}

1) Xie Guiyun ${ }^{[14]}$ recommended that the patient should gargle with sterile saline twice before receiving anesthesia and undergoing tracheal intubation. Gargling with saline solution can effectively decrease the number of bacteria in the mouth and during intubation, thus preventing bacterial colonization and decreasing the incidence of lower respiratory tract infection. 3) The results of a comparative study by Liu Chunxiang et al. ${ }^{[15]}$ showed that scrubbing the patient's mouth with cotton balls soaked in 3\% hydrogen peroxide solution followed by rinsing with saline twice a day could effectively clean the mouth. This method reduces 
the adhesion and growth of oropharyngeal bacteria, thus preventing the incidence of infection among patients receiving oral tracheal intubation. 3) Lu Yulin et al. ${ }^{[16]}$ believed that gargling with $0.1 \%$ povidone iodine and $0.12 \%$ chlorhexidine acetate could reduce the colonization of Gram-negative bacilli and diplococci in the oropharynx in patients receiving general anesthesia.

\section{Atomization inhalation}

\section{Selection of timing}

Selecting the optimal time for atomization inhalation can improve expectoration by patients, reduce the incidence of postoperative pulmonary complications, and shorten the time of extubation after surgery. 1) Zhou Xihong et al. ${ }^{[17]}$ reported that applying oxygen atomization before surgery under general anesthesia with tracheal intubation increases treatment and total effectiveness rates compared with applying oxygen atomization inhalation after surgery. In addition, the postoperative extubation and oxygen inhalation times are significantly reduced. 2) Lei Yong et al. ${ }^{[18]}$ reported that after abdominal surgery with general anesthesia, providing atomization inhalation to prevent lung infection has exact curative and satisfactory results.

\section{Selection of atomization inhalation device}

Li Liexia et al. ${ }^{[19]}$ compared and analyzed the effects of two kinds of post-operative atomization inhalation therapy on 125 cases of tracheal intubation. They found that the total effectiveness rate of the oxygen-driven atomization group is higher than that of the ultrasonic atomization group. Moreover, the $\mathrm{SaO}_{2}$ of the oxygen-driven atomization group is higher than that of the ultrasonic atomization group. In addition to its effectiveness, the oxygen-driven atomizer can be cleaned and disinfected thoroughly, and has a small size and simple preservation method; these characteristics prevent the spread of infection. Clinical studies by Yang Yanhong et al. ${ }^{[20]}$ showed that compression atomization inhalation after thoracotomy exerts better preventative effects against pulmonary complications than ultrasonic atomization inhalation.

\section{Selection of atomization inhalation}

Clinicians have no consensus on the optimal choice of atomization solution. $\alpha$-chymotrypsin, dexamethasone, and gentamicin are routinely used as atomization solutions. Wu Li-ping ${ }^{[21]}$ recommended ambroxol hydrochloride injection; Yan Gang ${ }^{[22]}$ recommended myrtle oil; Cheng Aibin et al. ${ }^{[23]}$ recommended budesonide suspension; and Gan Chuncheng recommended Tanreqing injection ${ }^{[24]}$. All of these atomization solutions have good effects.

\section{Personalized methods for sputum expectoration Spontaneous expectoration}

After atomization inhalation and percussion on the back, patients perform explosive or two-step expectoration on their own. The nurse stands beside the patient with her hands on both sides of the patient's incision. The nurse squeezes her hands to relieve the pain caused by the traction of the incision when the patient coughs, thus helping to strengthen the respiratory muscle of the patients ${ }^{[25]}$.

\section{Expectoration induced by irritation}

This method is suitable for patients with viscous sputum and the inability to expectorate. When the patient coughs, the nurse places their index and middle fingers on the tracheal position of the patient's sternum handle incisura to stimulate the cough reflex. With another hand, the nurse presses on the patient's abdomen to apply abdominal pressure until the cough stops, thus stimulating the expectoration of mucus attached to the trachea ${ }^{[26]}$.

\section{Expectoration via the respiratory scavenging system(VestTM System) method}

The VestTM system is a convenient airway removal device. This system consists of an inflatable vest connected by two pipes to a small pneumatic pulse generator that quickly inflates and deflates the vest. The vest can complete 20 cycles of soft compression and release on the chest wall per second. This process is high-frequency oscillatory ventilation, which can induce micro-coughs to promote the detachment of mucus from the bronchial wall, thus increasing its mobility, as well to promote the movement of the central airway. This process also acts on sticky secretions, facilitating their discharge. The expectoration effect and patient acceptance of the Vest TM system are superior to those of the traditional artificial percussion expectoration method ${ }^{[27]}$.

\section{On-demand aspiration of sputum}


If cleaning the respiratory tract is ineffective for some patients, sputum should be aspirated to minimize the retention of secretions. To address poor postoperative pulmonary function, patients with atelectasis should undergo fiber bronchoscopy for the targeted aspiration of sputum with high efficiency and limited damage ${ }^{[28]}$.

\section{Other methods}

\section{Semi-recumbent position in the early stage}

The patient should be placed in the semi-recumbent position as soon as possible after surgery under general anesthesia. The patient takes the head-up position, and the angle of the hospital bed should be gradually adjusted to $20^{\circ}-45^{\circ}$. This position is not only conducive to ventilation but also increases returned blood volume and cardiac output, thus promoting systemic circulation, improving blood oxygen content, and correcting systemic hypoxia. The semi-recumbent position can decrease tension and pain from the abdominal incision, consequently improving breathing. In addition, gastrointestinal decompression and unobstructed drainage can be maintained in the semirecumbent position, thus preventing the reflux of stomach contents through the stomach and esophageal sphincter into the throat ${ }^{[29]}$.

\section{Abdominal bandage compression binding}

After surgery, applying abdominal bandage compression binding with the appropriate degree of tightness can enhance the patients' effective thoracic breathing, which compensates for postoperative pain from the incision and abdominal breathing with low efficiency. Compression binding will also attenuate the patient's incision pain caused by coughing, allowing the patient to cough effectively with expectoration and deep breathing ${ }^{[30]}$.

\section{Reasonable analgesic use}

After surgery, analgesics should be provided to allow the patient to obtain adequate rest for the maintenance of physical strength and resistance to respiratory disease. Wang He et al. ${ }^{[31]}$ held that compared with intravenous selfcontrolled analgesia, epidural self-controlled analgesia after thoracic or abdominal surgery can significantly decrease the incidence of postoperative pneumonia, thus improving the patient's postoperative first-second forced expiratory volume and forced vital capacity.

\section{Judicious use of antibiotics}

Clinical studies have shown that isolates from cases of respiratory tract infection are mainly multi-drug resistantpathogens. Clinicians should judiciously use antimicrobial drugs based on the results of drug susceptibility tests ${ }^{[32]}$.

\section{Prevention of exogenous infection}

1) Hand hygiene is the most simple and effective control method for exogenous hospital infection. Zhang Xiaomei et al. ${ }^{[33]}$ reported that rapid hand disinfection with skin disinfectant exerts a better disinfection effect than hand washing with running water and hand sanitizer; these two disinfection methods have pass rates of $100 \%$ and $84 \%$. Skin disinfectant is easy applied and dries with good disinfection effect. 2) In each invasive operation, the principles of sterile operation must be strictly followed to strengthen the disinfection and sterilization of medical supplies. Disposable medical supplies should be used whenever possible.

\section{Environmental management}

Keep the indoor air and environment clean with regular air disinfections, window ventilation, and controlled ambient temperature. The number of accompanying staff should be restricted. The family members and medical staff of patients with respiratory diseases should not enter the ward. Indoor temperature should be maintained at $18{ }^{\circ} \mathrm{C}-22^{\circ} \mathrm{C}$ with humidity at $50 \%-60 \%$.

\section{Summary}

There are numerous reasons for respiratory tract infection after abdominal surgery under general anesthesia. Thus, the perioperative management of the respiratory tract should be improved. Health education, respiratory function training, oral care intervention, atomization inhalation, and personalized expectoration methods should receive sufficient attention. Clinicians must learn new theories, gain new knowledge, and update old concepts to decrease the incidence of complications and promote the early rehabilitation of patients after abdominal surgery. 


\section{Declarations}

\section{Acknowledgements}

No.

\section{Competing interests}

The author declares that she has no competing interest.

\section{Authors' contributions}

CX Yang made the literature analysis and wrote, discussed and revised the manuscript of this review.

\section{References}

1 Wang J, Zhao X. Analysis of related factors of postoperative lower respiratory tract infection and anesthesia and intervention strategies. Journal of Clinical Pulmonary Medicine, 2013, 18(7): 1262-1263.

2 Yang H, Yao X, Huang X. Investigation and analysis of postoperative pulmonary infection in patients with general anesthesia and tracheal intubation. Chinese Journal of Nosocomiology, 2009, 19(21): $2869-2870$

3 Bai X. Analysis of related factors of postoperative lower respiratory tract infection and nursing strategies. Journal of Henan Surgery, 2007, 13(3): 108-109.

4 Wei C, Liang Y, Li F. Risk factors of respiratory tract infection in patients with general anesthesia. Chinese Journal of Nosocomiology, 2011, 21(17): 3606-3607.

5 Duan H, Zhang Z, Fan J. Clinical analysis and nursing of 15 cases of general anaesthesia with difficult airway tracheal intubation. Clinical Medicine Practice, 2011, 20(3): 224-225.

6 Bao D. Postoperative nursing of respiratory tract of thoracic surgery patients. Chinese and foreign medical research, 2009, 7(13): 150-151.

7 Lin J, Chen M, Zhou X. Correlational study on hand hygiene compliance of medical workers and hospital infection. Chinese Journal of Nosocomiology, 2009, 19(2): 185-187.

8 Zhu H, Zhou C. Analysis of the factors of lower respiratory tract infection with general anesthesia and tracheal intubation. Chinese Journal of Nosocomiology, 2011, 21(18): 3832-3834.

9 Zhao W, Li L, Chen Y. Nursing intervention of 40 cases of pulmonary infection in elderly patients with mouth breath. Journal of Qilu Nursing, 2009, 15(21): 108-109.

10 Han H. Postoperative factors of lower respiratory tract infection and nursing strategies. Journal of Qilu Nursing, 2002, 8(6): 449-450.

11 Zhi X, Zha J, Liu L. The improvement of air quality in the respiratory ward by natural ventilation. Journal of Clinical Pulmonary Medicine,
2006, 11(6): 716-718.

12 Wang Y. Perioperative respiratory tract nursing of patients with general anesthesia laparotomy. Public Medical Forum Magazine, 2010, 14(36): 1152-1153.

13 Liu H, Xue C. Effect of preoperative respiratory function training on pulmonary function of patients with upper abdominal surgery. International Journal of Surgery, 2008, 35(2): 95-97.

14 Xie G. Study on the effectiveness of nursing intervention for lower respiratory tract infection caused by general anesthesia operation. Chinese Journal of Nosocomiology, 2012, 22(10): 2058-2059.

15 Liu C, Zhu L, Li D, et al. A comparative study on the effects of three nursing methods on oral care in patients with oral tracheal intubation. Journal of Nurses Training, 2013, 28(8): 677-679.

16 Lu Y, Yin J, Fan Ping, et al. Effect of preoperative oropharyngeal decontamination on oropharyngeal bacterial strains in general anesthesia patients. Journal of Nurses Training, 2011, 26(16): 1445-1447.

17 Zhou X, Lu W, Zhou X. Application of oxygen atomization inhalation before tracheal intubation anesthesia. Clinical Research, 2012, 10(33): $162-163$.

18 Lei Y, Zhao N. Clinical study of routine atomization inhalation after tracheal intubation with general anesthesia. Hebei medicine, 2012, 34(17): 2638-2639.

19 Li L, hang Y. Comparison of effects of the application of two atomization inhalation therapies. Journal of Qilu Nursing, 2013, 19(1): 121-122.

20 Yang Y, He Y. Comparison of two kinds of atomization inhalation treatments in postoperative pulmonary complications. Medical Aesthetics and Beauty, 2013, 9: 36-37.

21 Wu L. Observation of 50 cases of postoperative pulmonary complications prevented and treated by atomization inhalation of ambroxol hydrochloride and the nursing. China Pharmaceuticals, 2014, 23(1): $72-73$.

22 Yan Gang. Effect of atomization inhalation of myrtle oil on prevention and treatment of respiratory tract infection in patients with general anesthesia. China Practical Medicine, 2012, 7(5): 136-138.

23 Chen A, Shang X. Protective effect of inhaled budesonide suspension on airway in patients after general anesthesia operation. The Journal of Practical Medicine, 2013, 29(4): 675-676.

24 Gan C. The clinical study of the oxygen pneumatic atomization inhalation of Tanreqing injection in preventing postoperative respiratory tract infection after abdominal general anesthesia operation. Clinical Medicine, 2014, 27(1): 352-354.

25 An N, Yang C, Liu J, et al. Nursing progress of perioperative respiratory tract management in patients of general thoracic surgery. General Practice Nursing, 2011, 9(12): 3187-3189.

26 Yuan X, Ye Y. A review of postoperative methods of expectoration in patients with general anesthesia. Jiangxi Medicine, 2008, 43(11): 
1247-1248.

27 Gong Z, Zhang C, Li Xiang. Effect of respiratory tract clearance system on prevention of pulmonary infection in elderly patients after abdominal surgery. Chinese Journal of Modern Nursing, 2011, 17(23): 2765-2767.

, Chen D, Zhai R, et al. Analysis of clinical effect of fiberoptic bronchoscopy in the treatment of severe pulmonary infection. Chinese Journal of Modern Drug Application, 2014, 8(7): 100-101.

29 Li F. Observation and nursing of early semi-recumbent position and rehabilitation after abdominal operation with general anesthesia. Qinghai Medical Journal, 2009, 39(4): 32-33.

30 Sun S. Prevention and nursing of respiratory tract infections after abdominal operation with general anesthesia. The Chinese and Foreign Health Abstract, 2012, 9(41): 216-217.

31 Wang H, Sun X, Ma H. Meta-analysis of the effect of different controlled analgesia methods on postoperative complications of thoracic and abdominal surgery. Chinese Journal of Evidence-Based Medicine, 2010, 10(11): 1285 - 1292 .

32 Liu C, Zhu X, Sun Z, et al. Analysis of the distribution and drug resistance of isolates in respiratory tract secretion. Chinese Journal of Infection and Chemotherapy, 2011, 11(3): 219-223.

33 Zhang X, Liu X, Hu J. Different methods of hand washing effect monitoring of medical staff. Chinese Journal of Disinfection, 2013, 30(11): 1101-1102. 